\title{
Effect of citrus polyphenol- and curcumin-supplemented diet on inflammatory state in obese cats
}

\author{
Véronique Leray $^{1,3}$, Benjamin Freuchet ${ }^{1,3}$, Jérôme Le Bloc' ${ }^{1,3}{ }^{\text {, Isabelle Jeusette }}{ }^{2}$, Celina Torre $^{2}$ \\ and Patrick Nguyen ${ }^{1,3 *}$ \\ ${ }^{1}$ LUNAM Université, Oniris, National College of Veterinary Medicine, Food Science and Engineering, Nutrition and \\ Endocrinology Unit, Nantes F-44307, France \\ ${ }^{2}$ Affinity-Petcare, Saint Cugat (Barcelona), Spain \\ ${ }^{3}$ CRNH, Human Nutrition Research Center of Nantes, CHU, Nantes F-44093, France
}

(Received 28 October 2010 - Revised 30 March 2011 - Accepted 6 April 2011)

\section{Abstract}

Among obesity-associated disorders, low-grade inflammation has been described. The putative therapeutic properties of citrus and curcumin polyphenols could be associated with their anti-inflammatory properties. Two diets supplemented either with hesperidin (0.05\%) and naringin $(0.1 \%)$ from citrus extract or with highly bioavailable curcumin from Curcuma longa extract (0.09\%) were fed to eight obese cats for two 8-week periods (cross-over study design) while maintaining animals in an obese state. Plasma acute-phase protein (APP; $\alpha 1$-acid glycoprotein (AGP), serum amyloid A and haptoglobin) levels were assessed before and at the end of each test period. TNF- $\alpha$, IL-1 $\beta$, IL-2, IL-4, IL-5, IL-10, IL-12, IL-18, transforming growth factor- $\beta$, interferon (IFN)- $\gamma$ mRNA levels were determined in peripheral blood mononuclear cells (PBMC) by real-time PCR. Compared with pre-study values, supplementation with citrus polyphenols resulted in lower plasma AGP and haptoglobin concentrations, while that with curcumin resulted in lower plasma AGP concentration. There were no differences between the supplementations. TNF- $\alpha$, IL-1 $\beta$, IL- 4 , IL- 5 , IL-10, IL-12, IL-18, transforming growth factor- $\beta$, mRNA levels remained unaffected by either dietary supplementation. In contrast, IFN- $\gamma$ and IL- 2 mRNA levels were lower at the end of the citrus and the curcumin supplementation, respectively. There were no differences between the supplementations. The present study results show a slight effect of citrus and curcumin supplementation on inflammatory markers expressed by PBMC, and a decreased concentration of APP, which are mainly expressed by the liver. This would confirm that hesperidin and naringin or highly bioavailable curcumin extract have beneficial effects, targeted in the liver and could improve the obesity-related inflammatory state.

Key words: obesity: polyphenols: citrus: curcumin: inflammation: cat

The prevalence of obese or overweight cats is about 39\% of the feline population ${ }^{(1)}$. As obesity can predispose or exacerbate several serious medical conditions, its prevention or treatment is now a crucial issue. Indeed, obesity in cats is associated with hepatic lipidosis, dermatological disease, urinary tract disease and diabetes ${ }^{(2)}$. Moreover, chronic inflammation has been described in obese cats ${ }^{(3)}$.

In this context, any tool able to correct obesity or its effects would be useful, including a food supplement that could be effective in contributing to the loss of body fat mass and/or improving the inflammatory state of obese cats.

Plants synthesise numerous compounds, i.e. polyphenols, that may explain most of the beneficial health-related effects that have been reported with different plant extracts in rodents or with diets rich in fruits and vegetables in humans. Citrus and Curcuma longa are known sources of polyphenols.
In companion animals, citrus extract containing the polyphenols hesperidin and naringin has been shown to decrease plasma lipids and to have anti-inflammatory activity ${ }^{(4,5)}$. C. longa (turmeric) extracts, particularly the dietary polyphenol curcumin, have been shown to possess beneficial properties, especially anti-inflammatory properties ${ }^{(6)}$. Recent studies indicate that turmeric extracts are poorly absorbed following oral ingestion. However, a modified extraction process is known to enhance bioavailability of natural turmeric extract.

The present study aimed to determine the effects of hesperidin and naringin or highly bioavailable curcumin dietary supplements on plasma acute-phase protein (APP) concentration, and cytokine mRNA expression in isolated peripheral blood mononuclear cells (PBMC) from obese cats. The hypothesis was that such diets could specifically improve the inflammatory state of obese cats.

Abbreviations: AGP, $\alpha 1$-acid glycoprotein; APP, acute-phase protein; PBMC, peripheral blood mononuclear cells; SAA, serum amyloid A. 


\section{Materials and methods}

\section{Animals and diets}

European domestic shorthair neutered cats ( $n$ 8, three males, five females, 6.5 (SEM 0.5)-year old, mean body weight: 6.0 ( SEM 0.4) kg) were used in this study. They were classified as obese on the basis of their body condition score (mean 3.8 (sem 0.3), 1-5 possible) ${ }^{(7)}$. The cats had been obese for more than 1 year before the beginning of the study. Weight gain had originally been obtained by allowing ad libitum food consumption. All the cats were healthy based on physical examination and routine clinical laboratory data, except regarding obesity and associated insulin resistance. They were fed so as to maintain their obese body weight. The daily food quantity needed to reach this objective had been previously determined during the observation phase ( 4 weeks before the start of the study). Then, the cats were divided into two groups. Each group was fed a diet supplemented with either citrus extracts (protein 34.3\%, fat 드 $15.4 \%$, starch $30.6 \%$, hesperidin (Natural Orange Extract; Exquim SA, Barcelona, Spain) $0.05 \%$ and naringin (citroflavonoids soluble; Exquim SA) $0 \cdot 1 \%$ diet) or with highly bioavailable curcumin extract from C. longa (protein $34.2 \%$, fat $16.5 \%$, starch $28 \%$, Bio-Curcumin (BCM-95; Frutarom, Londerzeel, Belgium) $0.09 \%$ diet), for an 8 -week period, providing the same energy as was already determined as required to maintain body weight. Dietary inclusion level of polyphenols was chosen to reflect that typically recommended for human consumption. After this period, diets were switched and fed for another period of 8 weeks (cross-over design) after a 4-week washout period.

All animal handling procedures were carried out following the recommendations for animal welfare of the French Ministry of Agriculture. The experimental protocol adhered to European Union guidelines and was approved by the local Animal Use and Care Advisory Committee.

The plasma APP concentrations and the cytokine mRNA levels were assessed in isolated lymphocytes at the beginning of the trial and at the end of each 8-week period.

\section{Cytokine mRNA levels in isolated peripheral blood mononuclear cells}

Cells were isolated on Ficoll/Hypaque gradients. Total RNA was extracted using TRIzol reagent according to the manufacturer's protocol (Gibco BRL, Grand Island, NY, USA). After isolation, RNA pellets were dissolved in water and were then quantified spectrophotometrically. Total RNA ( $1 \mu \mathrm{g})$ was reverse transcribed in a $20-\mu l$ reaction volume using random primers (Pharmacia, Saclay, Orsay Cedex, France) and Superscript III Reverse Transcriptase (Invitrogen, Cergy Pontoise, France). After reverse transcription, $80 \mu \mathrm{l}$ distilled water was added.

cDNA solution $(2 \mu \mathrm{l})$ was subjected to real-time PCR conducted in an iCycler System (Bio-Rad, Hercules, CA, USA) using the MasterMix kit (Eurogentec, Angers, France). PCR consisted of a $10-\mathrm{min}$ denaturation at $95^{\circ} \mathrm{C}$ followed by 40 cycles $\left(15 \mathrm{~s}\right.$ at $95^{\circ} \mathrm{C}$ and $1 \mathrm{~min}$ at $60^{\circ} \mathrm{C}$ ). The sense/anti-sense primer sequences were designed using the web-based Primer3 program (http://frodo.wi.mit.edu/primer3/) and are shown in Table 1. The glyceraldehyde-3-phosphate dehydrogenase mRNA level was used as a reference value. Relative quantitative expression was calculated by the $2^{-\Delta \Delta C t}$ $\operatorname{method}^{(8)}$. The level of expression before the supplementation was arbitrarily set at $100 \%$.

\section{Plasma acute-phase protein concentrations}

Cephalic vein blood samples were collected from cats unfed overnight and centrifuged at $3000 \mathrm{~g}$ for $10 \mathrm{~min}$ at $4^{\circ} \mathrm{C}$. Plasma APP (serum amyloid A (SAA; haptoglobin and $\alpha 1$ acid glycoprotein (AGP)) were assayed using commercial ELISA kits (Abcys, Paris, France).

\section{Statistical procedures}

Data were checked for normality and equality of variance. For plasma peptide concentrations, comparison was performed using paired Student's $t$ test. For gene expression, comparison among means was performed by Wilcoxon test for nonparametric values. A $P$-value $<0.05$ was considered to be statistically significant. All statistical procedures were performed using StatView 5.0 software (SAS Institute, Cary, NC, USA).

\section{Results}

\section{Plasma acute-phase protein concentrations}

Table 1 shows the plasma concentrations of SAA, haptoglobin and AGP before (T0), and at the end of the citrus- (Citrus) or the curcumin-supplementation period (Curcumin). There was no effect of either supplement on plasma SAA concentrations, compared with the beginning of the study (T0). At the end of the citrus-supplementation period, plasma haptoglobin and

Table 1. Sense/anti-sense primers used for IL-1 $\beta$, IL-2, IL-4, IL-10, IL-12, IL-18, interferon (IFN)- $\gamma$, TNF- $\alpha$, transforming growth factor (TGF)- $\beta$ and glyceraldehyde-3-phosphate dehydrogenase (GAPdH) relative quantification

\begin{tabular}{|c|c|}
\hline Genes & Sense/anti-sense primers \\
\hline$I L-1 \beta$ & $\begin{array}{l}\text { 5'-AGTACCTGAACTCACCAGTG-3' } \\
5^{\prime} \text {-TAGTCCTGTGACTGTATGGC-3' }\end{array}$ \\
\hline IL-2 & $\begin{array}{l}5^{\prime} \text {-ACGGTTGCTTTTGAATGGAG-3' } \\
5^{\prime} \text {-CAATTCTGTGGCCTTCTTGG-3' }\end{array}$ \\
\hline$I L-4$ & $\begin{array}{l}5^{\prime} \text {-CCCCTAAGAACACAAGTGACAAG- } 3^{\prime} \\
5^{\prime} \text {-CCTTTGAGGAATTTGGTGGAG-3' }\end{array}$ \\
\hline IL-10 & $\begin{array}{l}5^{\prime} \text {-ACTTTAAGGGTTACCTGGGTTG-3' } \\
5^{\prime} \text {-CGTGCTGTTTGATGTCTGG-3' }\end{array}$ \\
\hline IL-12 & $\begin{array}{l}5^{\prime} \text {-GCCATTCGTTCCTCCTGATA-3' } \\
5^{\prime} \text {-GCAGGTGAAACGTCCAGAAT- } 3^{\prime}\end{array}$ \\
\hline IL-18 & $\begin{array}{l}\text { 5'-TTTGTAGCTGACAGTGATGAAAACC-3' } \\
5^{\prime} \text {-CAGGTTGATCTCCCTGGTTAATG-3' }\end{array}$ \\
\hline$I F N-\gamma$ & $\begin{array}{l}\text { 5'-CACCAAGATCTAACCTGAGGAAGC-3' } \\
\text { 5'-TATTGCAGGCAGGATGACCAT-3' }\end{array}$ \\
\hline$T N F-\alpha$ & $\begin{array}{l}\text { 5'-CACATGGCCTGCAACTAATC-3' } \\
\text { 5'-AGCTTCGGGGTTTGCTACTAC-3' }\end{array}$ \\
\hline$T G F-\beta$ & $\begin{array}{l}5^{\prime} \text {-CACGTGGAGCTGTACCAGAA- } 3^{\prime} \\
5^{\prime} \text {-GAGGCGAAAGCCCTCTACTT- } 3^{\prime}\end{array}$ \\
\hline$G A P d H$ & $\begin{array}{l}\text { 5'-GAGCTGAATGGGAAGCTCAC-3' } \\
\text { 5'-CGTATTTGGCAGCTTTCTCC-3' }\end{array}$ \\
\hline
\end{tabular}


AGP concentrations were lower compared with the initial values. At the end of the curcumin-supplementation period, plasma AGP concentration was lower, compared with T0 value. There were no significant differences in any of the plasma APP concentrations between the two supplementations (Table 2).

\section{Cytokine mRNA levels in isolated peripheral blood mononuclear cells}

Compared with the pre-study values (T0), cytokine mRNA levels were not different at the end of each period, except the interferon- $\gamma$ mRNA level that was lower at the end of the citrus-supplementation period (12 (SEM 3) v. 100 (SEM 40) $\%, P<0.05)$ and the IL-2 mRNA level that was lower at the end of the curcumin-supplementation period ( 48 (SEM 14) v. $100(\mathrm{SEM} 18) \%, P<0.05)$ than at T0. There were no significant differences in any of cytokine mRNA levels in isolated PBMC between the two supplementations.

\section{Discussion}

A chronic low-grade inflammatory state has been described in obese human subjects ${ }^{(9)}$ and dogs ${ }^{(10)}$. Many features associated with obesity could be involved in the development of an inflammatory state, even if the link between them is still not fully elucidated. First of all, because of the expansion of adipose tissue, adipocytes are more distant from blood vessels, and could therefore suffer from hypoxia which is associated with the release of cytokines ${ }^{(11)}$, and an alteration of the insulin signalling pathways that leads to a lower insulin sensitivity ${ }^{(12)}$. The attraction of macrophages to adipose tissue and their activation result in an amplification of local inflammation $^{(13,14)}$. The recruitment of $\mathrm{T}$ cells has been described as playing a fundamental role in the initiation and propagation of adipose tissue inflammation ${ }^{(15)}$, leading to a systemic inflammation that is correlated with insulin resistance.

In the present study, we aimed to assess the specific effects of citrus- or curcumin-supplemented diet on low-grade inflammation in obese cats. For this, obese cats were fed quantities of diet necessary to maintain them in an obese state, and we measured the APP levels in plasma and the expression of cytokine mRNA in isolated PBMC. In these conditions, the results showed a slight effect of citrus- and curcuminsupplemented diets on IL-2 and interferon- $\boldsymbol{\gamma}$ expression, respectively. There were no significant differences in any cytokine mRNA level between the citrus and the curcumin supplementations. In contrast, these diets seemed to lower APP levels in plasma, especially the citrus-supplemented diet after which haptoglobin and AGP concentrations were lowered. There were no differences in plasma APP concentrations between the citrus and the curcumin supplementation.

APP belongs to a class of plasma proteins, levels of which increase (positive APP) or decrease (negative APP) in response to inflammation. Haptoglobin, SAA and AGP are positive APP, associated with a chronic inflammatory state, they are synthesised mainly by the liver but also by other tissues such as adipose tissue ${ }^{(16,17)}$, and the plasma concentration that we measured in this study reflected therefore the secretion from both the liver and other tissues.

Hesperidin, naringin and curcumin belong chemically to the polyphenol family, and have been used for centuries for their biological activity. Curcumin is a spice derivative that has been reported to lower cytokine production in diabetic rats ${ }^{(18)}$, and to improve inflammatory state in a mouse model of obesity ${ }^{(19)}$. In the present study, we did not confirm these results. This could be explained at least in part by the difference between the doses of curcumin that animals received. In the present study we wanted to test concentrations that would reflect typically recommended consumption (curcumin 0.09\% diet), whereas in the previous in vivo studies, animals were fed diets with very high concentrations of curcumin $\left(3 \%{ }^{(19)}\right.$, $100 \mathrm{mg} / \mathrm{kg}$ body weight $\left.{ }^{(18)}\right)$. In the same way, citrus plant extracts have been shown to have anti-inflammatory activities in canine leucocytes ${ }^{(4)}$. However in that study, leucocytes were from dogs that were not obese, and the genes whose expression was altered by citrus extracts were different from the genes we studied here.

One study ${ }^{(20)}$ performed in mice has shown a lower plasma haptoglobin concentration in an adjuvant arthritis model pretreated with Curcuma. The present study is, at least to the best of our knowledge, the first one concerning the effects of dietary citrus or curcumin on concentration of APP responsible for obesity-associated inflammation in cats.

Two phenomena could explain why citrus and curcumin supplementation had no effect on cytokine expression by PBMC while they decreased APP concentrations in plasma. First, it is possible that the supplements, at least at the dose we chose, could be efficient in their action on the liver after their absorption, and in lowering hepatic synthesis of APP;

Table 2. Plasma acute-phase protein concentration before (TO) and at the end of feeding a citrus-supplemented diet (Citrus) and curcumin-supplemented diet (Curcumin) to obese cats*

(Mean values with their standard errors; $n$ 8)

\begin{tabular}{|c|c|c|c|c|c|c|c|c|}
\hline & \multicolumn{2}{|c|}{ T0 } & \multicolumn{3}{|c|}{ Citrus } & \multicolumn{3}{|c|}{ Curcumin } \\
\hline & Mean & SEM & Mean & SEM & $P$ & Mean & SEM & $P$ \\
\hline $\mathrm{SAA}(\mathrm{ng} / \mathrm{ml})$ & $2 \cdot 36$ & 0.14 & 3.79 & 0.64 & & $2 \cdot 74$ & 0.67 & \\
\hline Haptoglobin $(\mathrm{mg} / \mathrm{ml})$ & 1.52 & 0.12 & $1 \cdot 13$ & 0.11 & $<0.002$ & 1.30 & 0.08 & $<0.02$ \\
\hline AGP $(\mathrm{mg} / \mathrm{ml})$ & 524 & 66 & 251 & 31 & $<0.005$ & 320 & 23 & \\
\hline
\end{tabular}

SAA, serum amyloid A; AGP, $\alpha 1$-acid glycoprotein.

* Comparison was performed between Citrus and T0, and Curcumin and T0 by paired Student's $t$ test. There were no differences in plasma acute-phase protein concentrations between the citrus and the curcumin supplementation. 
whereas they could be inefficient in their action on blood cells, possibly because of a low concentration. Second, it is well established that mononuclear blood cells play an essential role in acute inflammation, whereas macrophages are central in chronic inflammation. Obesity-associated inflammation is clearly a chronic type. Thus, it is possible that mononuclear blood cells play a minor role in this context, and the citrus- and the curcumin-supplemented diet might improve inflammatory state in obese cats by acting on other inflammatory cells. These results are consistent with the fact that any intervention that leads to a decrease in chronic inflammation in obese subjects, such as weight loss ${ }^{(21)}$ or rosiglitazone treatment ${ }^{(22)}$, lowers circulating SAA levels. However, the small number of animals is an additional consideration. Indeed, for some parameters (TNF- $\alpha$, IL-4, IL-5, IL-10, IL-12), the observed numerical decrease could become significant by increasing the statistical power of the test.

The present study shows that citrus and curcumin supplementation lower plasma APP levels. This suggests, to our knowledge for the first time, an inflammatory state in obese cats that could be improved by dietary citrus and curcumin supplementation, especially through an action targeted on the liver.

\section{Acknowledgements}

V. L., I. J., C. T. and P. N. conceived, conceptualised and supervised the present study. V. L., J. L. B. and B. F. acquired and analysed data. The authors thank S. Ninet and P. Bleis for their technical assistance. All authors have contributed to the preparation of the manuscript and agree with the submitted manuscript content, and have no conflict of interest.

\section{References}

1. Courcier EA, O'Higgins R, Mellor DJ, et al. (2010) Prevalence and risk factors for feline obesity in a first opinion practice in Glasgow, Scotland. J Feline Med Surg 12, 746-753.

2. Scarlett JM \& Donoghue S (1998) Associations between body condition and disease in cats. J Am Vet Med Assoc 212, 1725-1731.

3. Lusby AL, Kirk CA \& Bartges JW (2009) The role of key adipokines in obesity and insulin resistance in cats. $\mathrm{J} \mathrm{Am} \mathrm{Vet}$ Med Assoc 235, 518-522.

4. Salas A, Subirada F, Pérez-Enciso M, et al. (2009) Plant polyphenol intake alters gene expression in canine leukocyte. J Nutrigenetics Nutrigenomics 2, 43-52.

5. Jeusette I, Torre C, Salas A, et al. (2010) Effects of consuming diets containing various fats or citrus flavanones on plasma lipid and urinary F2-isoprostane concentrations in overweight cats. Am J Vet Res 71, 1039-1044.

6. Aggarwal BB \& Harikumar KB (2009) Potential therapeutic effects of curcumin, the anti-inflammatory agent, against neurodegenerative, cardiovascular, pulmonary, metabolic, autoimmune and neoplastic diseases. Int J Biochem Cell Biol 41, 40-59.

7. Laflamme DP (1997) Development and validation of a body condition score system for cats: a clinical tool. Feline Pract 22, 13-18.

8. Livak KJ \& Schmittgen TD (2001) Analysis of relative gene expression data using real-time quantitative PCR and the 2(-Delta Delta C(T)) method. Methods 25, 402-408.

9. Monteiro R \& Azevedo I (2010) Chronic inflammation in obesity and the metabolic syndrome. Mediators Inflamm 2010, 289645-289655.

10. German AJ, Ryan VH, German AC, et al. (2010) Obesity, its associated disorders and the role of inflammatory adipokines in companion animals. Vet $J \mathbf{1 8 5}, 4-9$.

11. Ye J, Gao Z, Yin J, et al. (2007) Hypoxia is a potential risk factor for chronic inflammation and adiponectin reduction in adipose tissue of ob/ob and dietary obese mice. Am J Physiol Endocrinol Metab 293, E1118-E1128.

12. Regazzetti C, Peraldi P, Grémeaux T, et al. (2009) Hypoxia decreases insulin signaling pathways in adipocytes. Diabetes 58, $95-103$.

13. Weisberg SP, McCann D, Desai M, et al. (2003) Obesity is associated with macrophage accumulation in adipose tissue. J Clin Invest 112, 1796-1808.

14. Xu H, Barnes GT, Yang Q, et al. (2003) Chronic inflammation in fat plays a crucial role in the development of obesity related insulin resistance. J Clin Invest 112, 1821-1830.

15. Nishimura S, Manabe I, Nagasaki M, et al. (2009) $\mathrm{CD}^{+}$effector $\mathrm{T}$ cells contribute to macrophage recruitment and adipose tissue inflammation in obesity. Nat Med 15, 914-920.

16. Yang RZ, Lee MJ, Hu H, et al. (2006) Acute-phase serum amyloid A: an inflammatory adipokine and potential link between obesity and its metabolic complications. PLoS Med 3, 884-894.

17. Trayhurn P \& Wood IS (2004) Adipokines: inflammation and the pleiotropic role of white adipose tissue. BrJ Nutr $\mathbf{9 2}$, 347-355.

18. Jain SK, Rains J, Croad J, et al. (2009) Curcumin supplementation lowers TNF-alpha, IL-6, IL-8, and MCP-1 secretion in high glucose-treated cultured monocytes and blood levels of TNF-alpha, IL-6, MCP-1, glucose, and glycosylated hemoglobin in diabetic rats. Antioxid Redox Signal 11, 241-249.

19. Weisberg SP, Leibel R \& Tortoriello DV (2008) Dietary curcumin significantly improves obesity-associated inflammation and diabetes in mouse models of diabesity. Endocrinology 149, 3549-3558.

20. Tohda C, Nakayama N, Hatanaka F, et al. (2006) Comparison of anti-inflammatory activities of six curcuma rhizomes: a possible curcuminoid-independent pathway mediated by Curcuma phaeocaulis extract. Evid Based Complement Alternat Med 3, 255-260.

21. O'Brien KD, Brehm BJ, Seeley RJ, et al. (2005) Diet induced weight loss is associated with decreases in plasma serum amyloid A and C-reactive protein independent of dietary macronutrient composition in obese subjects. J Clin Endocrinol Metab 90, 2244-2249.

22. Mohanty P, Aljada A, Ghanim H, et al. (2004) Evidence for a potent antiinflammatory effect of rosiglitazone. J Clin Endocrinol Metab 89, 2728-2735. 